\title{
A new method for tuning PI controllers with Symmetric Send-On-Delta sampling strategy
}

\author{
Julio Ariel Romero Pérez*, Roberto Sanchis Llopis \\ Departament d'Enginyeria de Sistemes Industrials $i$ Disseny \\ Universitat Jaume I, Campus de Riu Sec \\ Castelló, Spain
}

\begin{abstract}
In this paper we present a new method for tuning PI controllers with Symmetric Send-On-Delta (SSOD) sampling strategy. First we analyze the conditions that produce oscillations in event based systems considering SSOD sampling strategy. The Describing Function is the tool used to address the problem. Once the conditions for oscillations are established, a new robustness to oscillation performance measure is introduced which entails with the concept of phase margin, one of the most traditional measures of relative stability in closed-loop control systems. Therefore, the application of the proposed robustness measure is easy and intuitive. Then the tuning method is developed to tune PI controllers to avoid the limit cycles. Because the method is based on the Describing Function, the effect of higher order harmonics has been studied to evaluate the validity of the proposal. Unlike some previous works concerning SSOD based PI, the results presented in this article are not restricted to specific model structures, instead they can be applied to a wide range of linear processes including dead-time, non-minimum phase and under-damped response. The tuning method is tested in simulations with a batch of models widely used in PI design methods testing. Furthermore, an actual laboratory scale application is presented to prove the feasibility of the proposal. Additionally, a Java application has been developed to aid in the design of SSOD based PI controllers according to the results presented in this paper.
\end{abstract}

Keywords:

PID controller, event-based system, tuning method

\footnotetext{
*Corresponding author

Email addresses: romeroj@uji.es (Julio Ariel Romero Pérez ), rsanchis@uji.es (Roberto Sanchis Llopis )
}

Preprint submitted to ISA Transactions

May 26, 2016 


\section{Introduction}

During the last years many researches have been focused in event based controllers as alternative to the classical time driven control systems. The main goals in the design of these controllers is the reduction of the frequency of measurement needed for control without degrading the closed loop performance. This is a basic requirement for controllers in networked control systems where many devices (sensors, actuators, controllers) share a communication channel with limited bandwidth. The reduction in the number of transmitted messages improve the network overall behavior, for example avoiding dropouts and delays. The use of wireless communications in control application has also encouraged the development of event based controllers. In this case the reduction of data transmission imply an important decrease in power consumption, therefore increasing the lifetime of batteries of self-powered remote sensors [1].

In this context several papers have been published where Event-Based PID control algorithms are proposed. These algorithms exploit the properties of the PID control while reducing the network traffic between the controller and the sensor using, in most of the cases, a sampling strategy based on the crossing of levels or thresholds $(\delta)$ by the control error signal. This strategy is known as Send-On-Delta (SOD), [2], and its effectiveness in controlling and reducing communication load has been widely contrasted $[3,4]$.

One of the first contributions on Event-Based PID control was introduced by Årzén [5] as a way to reduce the use of CPU in embedded control systems without significantly affecting the closed loop performance. To achieve this goal, the sensor is sampled periodically but the control algorithm is activated only if the error exceeds a given threshold. In that paper, some main issues in event-based PID control were addressed, such as the error in the calculation of the integral and derivative terms when the time between samples increases. Some further works have been focused on solving the problems raised by Årén, mainly related with the calculation of the integral error cited above. It should be noted in particular the works of Durand $[6,7]$ and Vasyutynskyy [8, 9].

A very simple variant of SOD in the context of event based PI controllers has recently been proposed by Beschi et al. in [10]: the sampled signal is quantified by a quantity multiple of $\delta$ so that the relationship between the input and output of the event-generator is symmetric with respect to the origin. Thus, this strategy is named symmetric send-on-delta (SSOD) sampling. 
Relevant results in the field of event based PI controller with SSOD sampling have been obtained during the last years. These results include: 1) necessary conditions on system instability and necessary and sufficient conditions on the controller parameters for the existence of equilibrium points without limit cycles for first order processes plus time delay (FOPTD)[10], 2) characterization of equilibrium point for different kind of systems such as integrating processes plus time delay (IPTD), first order processes plus time delay (FOPTD), and second-order processes plus time delay (SOPTD)[11], 3) tuning rules when the system has a FOPTD model [12], 4) auto-tuning method when the system has an integrator [13]. More general results about this kind of systems were presented in [14], where the authors established the conditions for the DC gain of the open loop transfer function which ensured the absence of oscillations, without assuming any specific model structure.

The main result presented in this paper is a new method for tuning PI controllers when SSOD is used in the control loop. First we analyze the conditions that produce oscillations in event based systems considering SSOD sampling strategy. Once the conditions for oscillations are established, a new robustness to oscillation performance measure is introduced, which entails with the concept of phase margin, one of the most traditional measures of relative stability in closed-loop control systems. Our proposal is based on the describing function (DF) technique, a well-known tool in the area of non-linear control systems. Because the DF is based on the assumption of negligible higher order harmonics in the close-loop system, which may be a strong assumption, a detailed study is presented here about effect of those harmonics in the proposed tuning method.

It is worth mentioning that the results presented in this article are not restricted to specific model structure, instead they can be applied to a wide range of linear processes including dead-time, non-minimum phase and under-damped response. All the theoretic results have been put together into a Java application to aid in the design of SSOD based PI controllers. The approach was tested by simulations, using

an extensive batch of process models, as well as the real application to a laboratory scale process.

\section{Problem statement}

Consider the networked control system shown in Figure 1, where $C(s)$ and $G(s)$ are the controller and the process transfer functions respectively, $y_{r}$ is the reference signal to be tracked, $y$ is the controlled output, and $p$ is the disturbance input. It is supposed that the controller is located near the actuator and the sensor sends measurements of process output $y$ (or more precisely of the tracking error $e$ ) to 
the controller through a communication network using the SSOD strategy. The $\mathrm{ZOH}$ block keeps in $\bar{e}$ the last sent value of process output $e^{*}$ until a new value is transmitted by the SSOD block.

Concerning the network behavior, it is assumed that the delays in packages transmission are either negligible or known and constant with value $t_{d}$. Although this may be considered restrictive for most real applications, there are powerful reasons to justify this assumption. First, most of industrial communication networks are fast enough to fulfill the timing requirements for process control applications where the system dynamics are slow in comparison to networks transmission rates. Second, nowadays a great research effort is being spent to design protocols to significantly reduce the package's collision, main cause of variable delays in communication networks, [15]. Communication delays are represented by the terms $\exp \left(-t_{d} s\right)$ in Figure 1 .

This control scheme was first proposed in [10] considering $C(s)$ a PI controller, so the authors called it SSOD-PI architecture. As commented in the introduction, some guide lines for the controller tuning were given in [12] when the process $G(s)$ is a FOPTD model. Our goal in this paper is to design the controller $C(s)$ in order to reach an adequate performance and robustness of the closed-loop system, for processes with known transfer functions $G(s)$ considering not only FOPTD models but also a more wide range of model structures. The objective of the tuning procedure is not only to guarantee stability but also to avoid persistent oscillations or limit cycles that can appear due to the SOD strategy if the controller is not designed properly.

Remark: Another possible send on delta scheme, called PI-SSOD in the literature, could also be considered. It consists of assuming that the controller is located near the sensor, and the SSOD policy is applied to the control action (that is sent to the actuator only when it changes more than delta). The drawback of this approach, as demonstrated by [10], is that the simple existence of the integrator leads to an oscillatory behavior unless one of the delta thresholds coincide with the exact input needed in steady state to maintain the output in the required setpoint. As this is not a realistic assumption in real applications, the PI-SSOD scheme always produce persistent oscillations, no matter how the controller is tuned. The only way to avoid oscillations in that case (see [10]) is to include a dead band in the error signal. That dead band would change the behavior of the original SOD approach and would make the direct application of the proposed describing function analysis not valid. For those reasons the study of the PI-SSOD scheme will be left for future works, and will not be considered in this paper. 


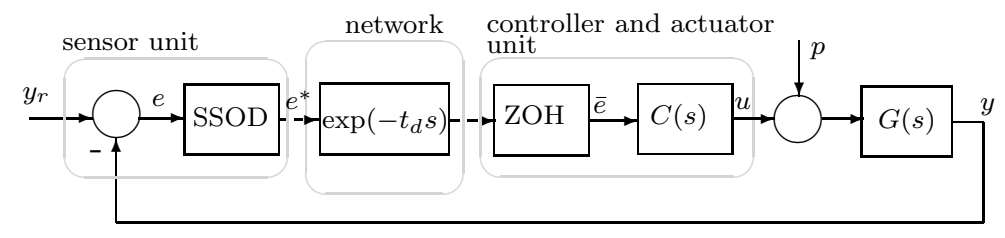

Figure 1: Networket control system with SSOD sampling strategy.

\section{Describing function based approach}

In the control scheme presented in Figure 1 the SSOD block is followed by a ZOH block. Figure 2 shows the relation between the SSOD input $e$ and the $\mathrm{ZOH}$ output $\bar{e}$. The small circles over the diagonal dotted line correspond to the values of $e^{*}$ that are sent to the $\mathrm{ZOH}$ block. A new value $e^{*}=i \delta, i \in \mathbb{Z}$ is sent to the $\mathrm{ZOH}$ block when $e$ crosses the levels $i \delta$. The ZOH block keeps this value in the output $\bar{e}$ until a new value $e^{*}$ is sent from the SSOD block. Horizontal continuous lines crossing the small circles highlight the fact that $\bar{e}$ keeps its value for variations of $\pm \delta$ around the $i \delta$ levels. In summary, $e^{*}$ denote the discrete values that are sent through the network when the error crosses the thresholds. On the other hand, $\bar{e}$ is a continuous signal that has the form of consecutive steps that changes when a value of $e^{*}$ is received.

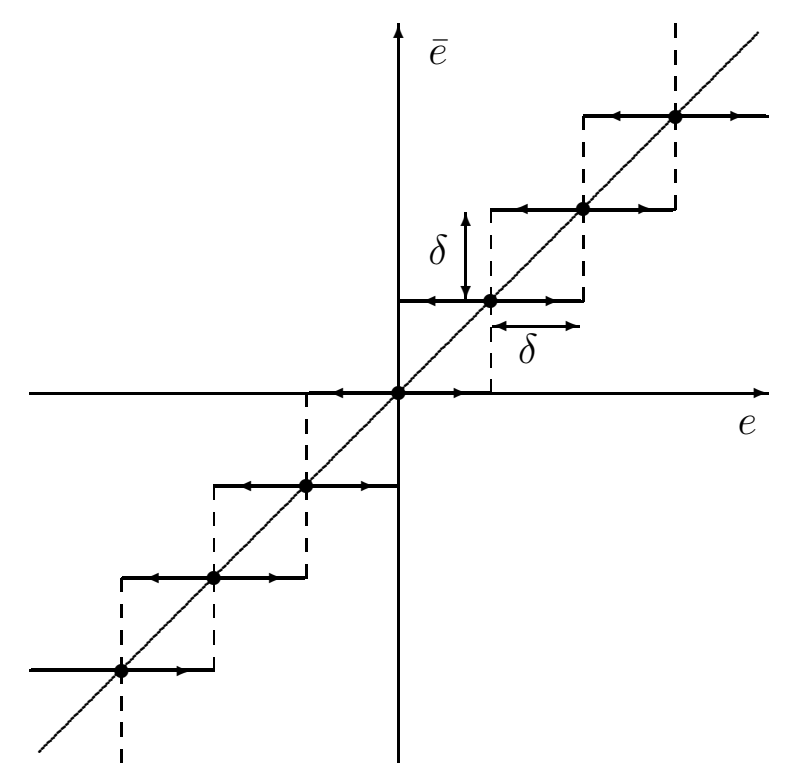

Figure 2: Input/Output characteristic of the SSOD_ZOH blocks combination.

According to the previous analysis, it is clear that the combination of SSOD 
and $\mathrm{ZOH}$ blocks results in a non-linearity. Consequently, the system in Figure 1, can be represented as that in Figure 3 where the block SSOD_ZOH represents the combination of SSOD and ZOH blocks. Therefore, the original problem of event based controller is transformed to a non-linear control problem that involves a linear system with a nonlinear block.

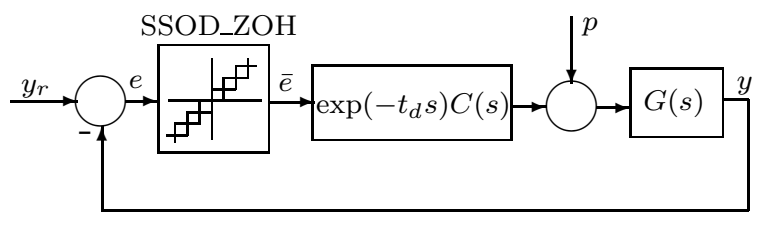

Figure 3: Non-linear equivalent system to the control systems with SSOD sampling strategy in Figure 1.

Following [16] it can be proved (see Appendix A) that the describing function of the SSOD_ZOH block in Figure 3, considering a sinusoidal input of amplitude $A$ is given by the equation (1),

$$
\begin{aligned}
N(A, \delta)= & \frac{2 \delta}{\pi A}\left[1+\sqrt{1-\left(m \frac{\delta}{A}\right)^{2}}\right. \\
& \left.+2 \sum_{k=1}^{m-1} \sqrt{1-\left(k \frac{\delta}{A}\right)^{2}}\right]-j m \frac{2 \delta^{2}}{\pi A^{2}}
\end{aligned}
$$

where

$$
m=\left\lfloor\frac{A}{\delta}\right\rfloor
$$

The describing function in equation (1) depends on the quotient $\frac{\delta}{A}$. According to Figure 2, the non-linearity output is zero for inputs with amplitude $A<\delta$, thus the quotient $\frac{\delta}{A}$ is limited to the interval $[0,1]$ and can be considered as a normalized parameter, $\delta_{A}$. Under this consideration the equation 1 can be written as:

$$
\begin{aligned}
N\left(\delta_{A}\right)= & \frac{2 \delta_{A}}{\pi}\left[1+\sqrt{1-\left(m \delta_{A}\right)^{2}}\right. \\
& \left.+2 \sum_{k=1}^{m-1} \sqrt{1-\left(k \delta_{A}\right)^{2}}\right]-j \frac{2 m \delta_{A}^{2}}{\pi}
\end{aligned}
$$


This DF can be used to analyze the existence of limit cycles in the closed loop system shown in Figure 3. The condition for the existence of limit cycles is given by the following equation [16]:

$$
G_{o l}(j \omega)=-\frac{1}{N\left(\delta_{A}\right)}
$$

where $G_{o l}(j \omega)$ is the open loop transfer function:

$$
G_{o l}(j \omega)=C(j \omega) G(j \omega) \exp \left(-t_{d} j \omega\right)
$$

Condition (3) corresponds to the intersections of $G_{o l}(j \omega)$ and $-1 / N\left(\delta_{A}\right)$ in the polar plot. The portrait of $-1 / N\left(\delta_{A}\right)$, which is shown in Figure 4 , can obtained by evaluating equation (2) for $\delta_{A} \in[0,1]$.

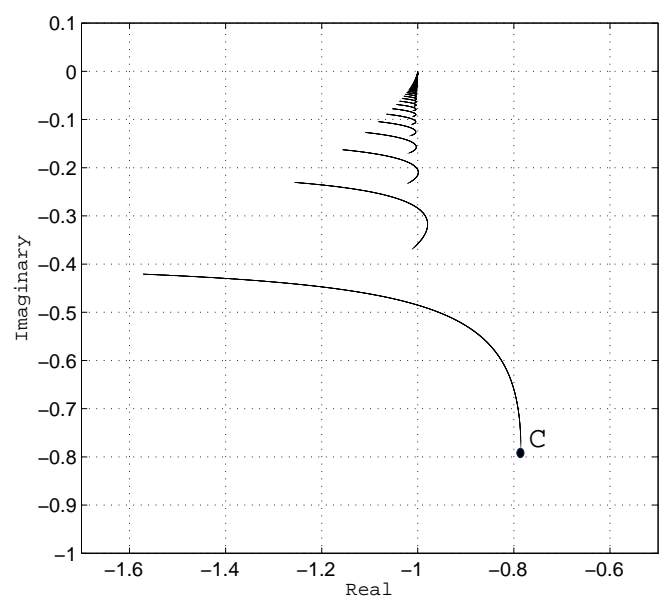

Figure 4: Polar plot of $-1 / N$.

\section{Tuning SSOD based PI controllers}

Taking into account the shape of the Nyquist diagrams of usual processes, the most critical point in the plot in order to avoid the intersection between $G_{o l}(j \omega)$ and $-1 / N\left(\delta_{A}\right)$ is the point $-\frac{\pi}{4}-\frac{\pi}{4} j=\frac{\pi \sqrt{2}}{4} \exp \left(-\frac{3 \pi}{4} j\right)$, shown as point $\mathrm{C}$ in Figure 4. This point is obtained for $\delta_{A}=1$. As it will be shown in the next section, for most of the common types of processes, if the Nyquist plot of $G_{o l}(j \omega)$ does not encircle 
that point, then there is no intersection with the DF plot, and hence, there are no oscillations.

Based on this simple idea, a new robustness measure is proposed to evaluate the robustness of the controlled system with respect to undesired oscillations: the SSOD phase margin, $\Phi_{m, s s o d}$, i.e. a phase margin to avoid limit cycle. It is defined as the phase that should be added to $G_{o l}(j \omega)$ to make the Nyquist plot cross the critical point $\mathrm{C}$, see Figure 5. To define this specification precisely, let us define a new gain crossover frequency, $\omega_{g^{\prime}}$, as the frequency where $\left|G_{o l}\left(j \omega_{g^{\prime}}\right)\right|=\frac{\pi \sqrt{2}}{4} \approx 1.11$. This new gain crossover frequency is different from the usual one, $\omega_{g}$, defined as $\left|G_{o l}\left(j \omega_{g}\right)\right|=1$. The phase margin to avoid limit cycle is then defined as:

$$
\begin{aligned}
\Phi_{m, \text { ssod }} & =\arg \left(G_{o l}\left(j \omega_{g^{\prime}}\right)\right)-\arg \left(-\frac{\pi}{4}-\frac{\pi}{4} j\right) \\
& =\arg \left(G_{o l}\left(j \omega_{g^{\prime}}\right)\right)+\frac{3 \pi}{4}
\end{aligned}
$$

It should be noted that the final phase margin in the classical sense, that measures robustness to instability, will be somehow near $\Phi_{m, s s o d}+\frac{\pi}{4}$ ( or $\left.\Phi_{m, s s o d}+45^{\circ}\right)$. However, it is not sufficient to choose a classical phase margin larger than $45^{\circ}$ to guarantee the avoidance of the limit cycle as the classical phase margin is defined where the magnitude is 1, and not 1.11, and hence, the Nyquist plot could still intersect the DF plot. The selection of the final phase margin greater than $45^{\circ}$, as proposed by the authors in [17], can be considered a general and intuitive rule of thumb for tuning SSOD based PI controllers, but not an exact method to avoid limit cycles as the one presented here.

Based on the definition of phase margin to oscillations $\left(\Phi_{m, s s o d}\right)$, the PI design method described in [18] is proposed to be modified in the following to be adapted to the SSOD sampling scenario. The transfer function of the PI controller is assumed to be

$$
C(s)=K_{p}\left(1+\frac{1}{T_{i} s}\right)=K_{p}+\frac{K_{i}}{s}
$$

where

$$
K_{i}=\frac{K_{p}}{T_{i}}
$$

The objective of the PI controller to be designed is to reach an adequate performance and robustness of the controlled system, taking into account the assumed event based sampling, expressed in the following design specifications:

- Required phase margin to avoid limit cycle: $\Phi_{m, s s o d, r}$. The phase margin $\left(\Phi_{m, s s o d}\right)$ should be equal to this value $\left(\Phi_{m, s s o d}=\Phi_{m, s s o d, r}\right)$. 


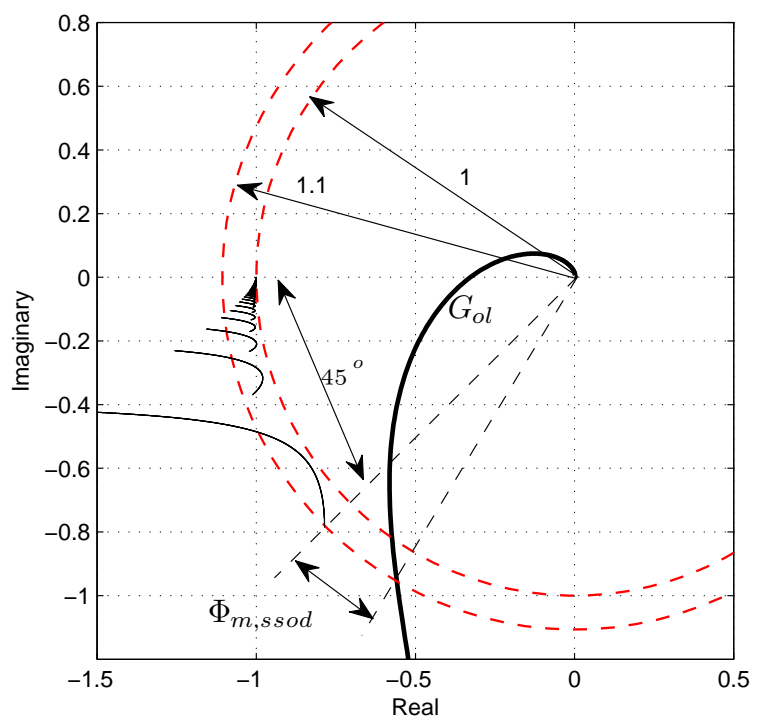

Figure 5: The new robustness measure, $\Phi_{m, s s o d}$

- Minimum required gain margin: $\gamma_{m, r}$. The gain margin $\left(\gamma_{m}\right)$ should be larger than or equal to this value $\left(\gamma_{m} \geq \gamma_{m, r}\right)$.

- Good disturbance rejection. The step disturbance IAE should be as low as possible.

\subsection{PI design method}

The design method can be summarized as a constrained optimization approach in which the proposed PI parameters are those that maximize the controller integral gain $\left(K_{i}=\frac{K_{p}}{T_{i}}\right)$, subject to the following constraints: $\Phi_{m, s s o d}=\Phi_{m, s s o d, r}, \gamma_{m} \geq \gamma_{m, r}$. The maximization of $K_{i}$ is equivalent to the minimization of the integral of the error (IE) of the step disturbance response, because it is well known that

$$
I E=\lim _{t \rightarrow \infty} \int_{0}^{t} e(\tau) d \tau=\lim _{s \rightarrow 0} \frac{G(s)}{s(1+C(s) G(s))}=\frac{1}{K_{i}}
$$

If the phase and gain margins specifications are large enough, then the closed loop response is not too oscillatory, and the IE is similar to the IAE. Therefore, the approach is equivalent to an approximate minimization of the IAE. 
The key to solve the previous constraint maximization problem straightforwardly is to introduce a tuning parameter, that is the relation between the final gain crossover frequency of the process with controller to avoid limit cycles and the controller zero

$$
a=\frac{\omega_{g^{\prime}}}{z_{i}}=\omega_{g^{\prime}} T_{i}
$$

where $\omega_{g^{\prime}}$ is the frequency where the magnitude of the open loop frequency response of the process plus controller is $G_{o l}\left(j \omega_{g^{\prime}}\right)=\frac{\pi \sqrt{2}}{4} \approx 1.11$, i.e. where the phase margin to avoid limit cycle is measured.

For a given value of this parameter $a$, the phase of the controller at the final crossover frequency $\omega_{g^{\prime}}$ depends only on that value:

$$
\arg \left(C\left(j \omega_{g^{\prime}}\right)\right)=\arctan (a)-\frac{\pi}{2}=-\arctan \left(\frac{1}{a}\right)
$$

Therefore, for a given value of parameter $a$, the calculation of the controller is automatic, following two steps

1. The final crossover frequency $\left(\omega_{g^{\prime}}\right)$ is obtained as the frequency where the phase of the system fulfills the phase margin equation, i.e. where the phase of the system is

$$
\arg \left(G\left(j \omega_{g^{\prime}}\right)\right)=-\frac{3 \pi}{4}+\Phi_{m, s s o d, r}-\arg \left(C\left(j \omega_{g^{\prime}}\right)\right)=\Phi_{m, s s o d, r}-\frac{\pi}{4}-\arctan (a)
$$

Then, the value of the integral time $\left(T_{i}\right)$ is calculated as

$$
T_{i}=\frac{a}{\omega_{g^{\prime}}}
$$

2. The value of $K_{p}$ is calculated from the condition of magnitude $\frac{\pi \sqrt{2}}{4} \approx 1.11$ at the final crossover frequency

$$
\left|C\left(j \omega_{g^{\prime}}\right) G\left(j \omega_{g^{\prime}}\right)\right|=\frac{\pi \sqrt{2}}{4} \rightarrow K_{p}
$$

The resulting equation for $K_{p}$ is:

$$
K_{p}=\frac{\pi \sqrt{2}}{4} \frac{a}{\left|G\left(j \omega_{g^{\prime}}\right)\right| \sqrt{1+a^{2}}}
$$


In the previous steps, the available frequency response data of the process is used, interpolating, if necessary, between the available points. Once the controller parameters are defined, the final gain margin, $\gamma_{m}$, can be calculated.

The proposed PI design method can be expressed as the following optimization problem over a single real parameter, $a$ :

$$
\begin{aligned}
& \max _{a} \quad K_{i} \\
& \text { s.t. } \quad \Phi_{m, s s o d}=\Phi_{m, s s o d, r} \\
& \gamma_{m} \geq \gamma_{m, r}
\end{aligned}
$$

This is a very simple one-dimensional optimization problem in which the search space for parameter $a$ is small and well defined. According to equation (9), the phase contribution of $\arg \left(C\left(j \omega_{g^{\prime}}\right)\right.$ ), is significant only in the range $0<a<6$ as shown in the Figure 6, therefore the optimal value of $a$ is expected to be in that range.

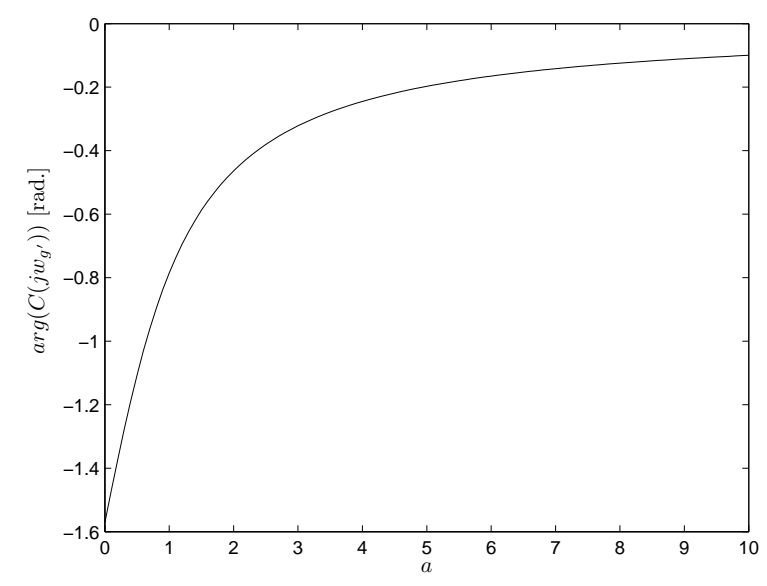

Figure 6: $\arg \left(C\left(j \omega_{g^{\prime}}\right)\right)$ as function of the parameter $a$.

Remark: The shape of $-\frac{1}{N\left(\delta_{A}\right)}$ in Figure 4 is completely defined by $\delta_{A} \in[0,1]$ and does not dependent on the value of $\delta$. That means that once the controller $C(s)$ is designed to avoid the limit cycle, this condition is fulfilled regardless of the value of the parameter $\delta$. As a result, nor stability neither robustness of the closed loop system in Figure 1 are affected by the value on $\delta$. The value of $\delta$, however, determines the steady state error because the block ZOH_SSOD in Figure 1 introduces a deadzone effect of amplitude $\delta$. Thus, greater values of $\delta$ may potentially lead to higher 
steady state errors. The parameter $\delta$ is also related to the event generation rate by an inverse relationship, [12]. Taking this into account, the selection of $\delta$ should be a trade-off between the steady state requirements and communication restrictions.

Remark: The proposed design approach does not take into account the use of a weighting factor for the proportional term of the reference. This weighting factor $b<1$ is usually added in the calculation of the proportional part of the control action as $u_{p}=K_{p}\left(b y_{r}-y\right)$. If the overshoot of the step reference response is too high, an adequate selection of this factor $b$ can reduce it to a lower level. Trial and error can be used to adjust the value of $b$. If a transfer function model of the process is available, this trial and error can be made in simulations.

\section{Validity of the approach}

The oscillation condition used to define the new phase margin was obtained by the DF method, which is valid only if the linear part of the system in Figure 3 is filtering enough to neglect the higher order harmonics in the input of the non-linearity. The relaxation of this condition could introduce errors in the limit cycle prediction, and might result in undesired oscillation. In this section we study the effect of the higher harmonics in the phase margin for oscillation $\Phi_{m, s s o d}$ predicted by de DF. Concretely we analyze the variations in the critical point $\mathrm{C}$ in Figure 4 due to these harmonics.

Suppose we design the PI that maximize the gain $K_{i}$ with $\Phi_{m, s s o d}=0$. With that controller, the open loop transfer function of the system in Figure 3 crosses the point C. Let us define $\omega_{c}$ as the frequency where $G_{o l}\left(j \omega_{c}\right)=-\frac{\pi}{4}-\frac{\pi}{4} j$. The resulting closed loop oscillation with that controller would be as shown in Figure 7, which shows the input $(e)$ and output $(\bar{e})$ of the non-linearity SSOD_ZOH. The signal $e$ fulfills conditions (14), where $T_{o}$ is the period of oscillation and the initial time is considered in the rising edge of $\bar{e}$. The frequency of oscillation, $\omega_{o}=2 \pi / T_{o}$, will be closed to $\omega_{c}$, but not exactly that one due to the higher order harmonics effects.

$$
\begin{gathered}
e\left(\frac{T_{o}}{4}\right)=0 \\
e\left(\frac{T_{o}}{2}\right)=-\delta
\end{gathered}
$$

The Fourier transform of $\bar{e}$ is:

$$
\bar{e}(t)=\frac{4 \delta}{\pi} \sum_{n=1,3,5, . .}^{\infty} \frac{1}{n}\left(\sin \left(\frac{n \pi}{4}\right) \cos \left(n w_{o} t-\frac{n \pi}{4}\right)\right)
$$




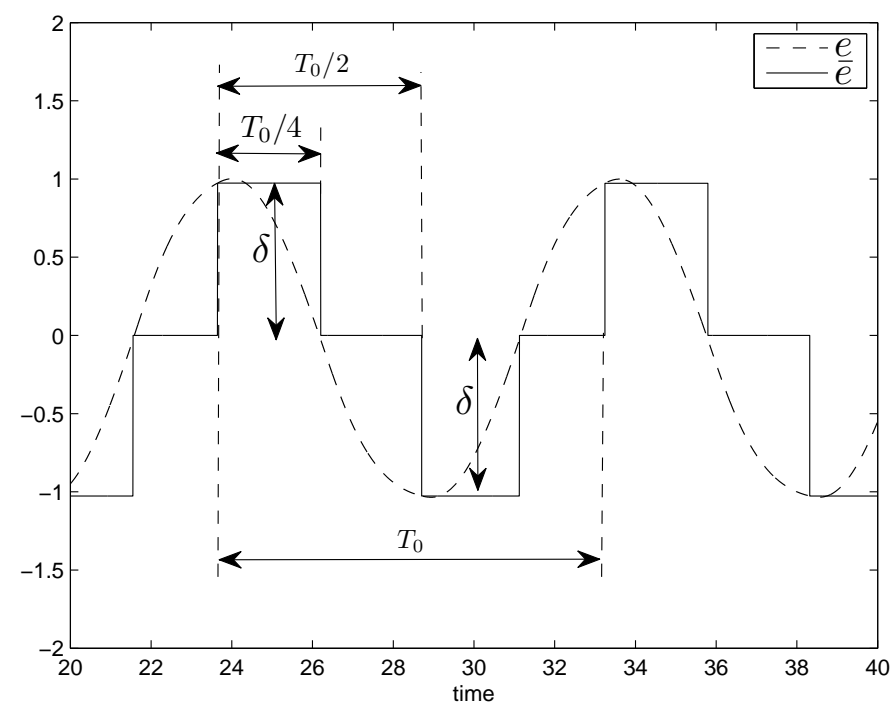

Figure 7: Oscillation in the SSOD PI based systems.

$$
\bar{e}(t)=\frac{4 \delta}{\pi} \sum_{n=1,3,5, . .}^{\infty} \frac{1}{n} \sin \left(\frac{n \pi}{4}\right) \text { real }\left(\exp \left(-j \frac{n \pi}{4}+j n w_{o} t\right)\right)
$$

where $w_{o}$ is the frequency of oscillation.

Then, the output of the open loop transfer function $G_{o l}(s)$ is:

$$
y(t)=\frac{4 \delta}{\pi} \sum_{n=1,3,5, . .}^{\infty} \frac{1}{n} \sin \left(\frac{n \pi}{4}\right) \operatorname{real}\left(G_{o l}\left(j n w_{o}\right) \exp \left(-j \frac{n \pi}{4}+j n w_{o} t\right)\right)
$$

Considering $y_{r}=0$, then $e(t)=-y(t)$, and evaluating $e(t)$ in $t=T_{0} / 4$ and $t=T_{0} / 2$ results in:

$$
\begin{aligned}
& e\left(T_{0} / 4\right)=-\frac{4 \delta}{\pi} \sum_{n=1,3,5, . .}^{\infty} \frac{1}{n} \sin \left(\frac{n \pi}{4}\right) \text { real }\left(G_{o l}\left(j n w_{o}\right) \exp \left(j \frac{n \pi}{4}\right)\right) \\
& e\left(T_{0} / 2\right)=\frac{4 \delta}{\pi} \sum_{n=1,3,5, . .}^{\infty} \frac{1}{n} \sin \left(\frac{n \pi}{4}\right) \operatorname{real}\left(G_{o l}\left(j n w_{o}\right) \exp \left(-j \frac{n \pi}{4}\right)\right)
\end{aligned}
$$

Defining 


$$
R_{o l}\left(n w_{o}\right)=\operatorname{real}\left(G_{o l}\left(j n w_{o}\right)\right) ; I_{o l}\left(n w_{o}\right)=\operatorname{imag}\left(G_{o l}\left(j n w_{o}\right)\right)
$$

and using the Euler expressions of the complex exponentials, then equations (18) and (19) can be written as

$$
\begin{gathered}
\left.e\left(T_{0} / 4\right)=-\frac{4 \delta}{\pi} \sum_{n=1,3,5, . .}^{\infty} \frac{1}{n} \sin \left(\frac{n \pi}{4}\right)\left(R_{o l}\left(n w_{o}\right) \cos \left(\frac{n \pi}{4}\right)-I_{o l}\left(n w_{o}\right) \sin \left(\frac{n \pi}{4}\right)\right)\right) \\
\left.e\left(T_{0} / 2\right)=\frac{4 \delta}{\pi} \sum_{n=1,3,5, . .}^{\infty} \frac{1}{n} \sin \left(\frac{n \pi}{4}\right)\left(R_{o l}\left(n w_{o}\right) \cos \left(\frac{n \pi}{4}\right)+I_{o l}\left(n w_{o}\right) \sin \left(\frac{n \pi}{4}\right)\right)\right)
\end{gathered}
$$

Therefore, the conditions expressed in (14) can be written as

$$
\begin{aligned}
& -\frac{4 \delta}{\pi} \sum_{n=1,3,5, . .}^{\infty} \frac{1}{n} \sin \left(\frac{n \pi}{4}\right) \cos \left(\frac{n \pi}{4}\right) R_{o l}\left(n w_{o}\right)+\frac{4 \delta}{\pi} \sum_{n=1,3,5, . .}^{\infty} \frac{1}{n} \sin ^{2}\left(\frac{n \pi}{4}\right) I_{o l}\left(n w_{o}\right)=0 \\
& \frac{4 \delta}{\pi} \sum_{n=1,3,5, . .}^{\infty} \frac{1}{n} \sin \left(\frac{n \pi}{4}\right) \cos \left(\frac{n \pi}{4}\right) R_{o l}\left(n w_{o}\right)+\frac{4 \delta}{\pi} \sum_{n=1,3,5, . .}^{\infty} \frac{1}{n} \sin ^{2}\left(\frac{n \pi}{4}\right) I_{o l}\left(n w_{o}\right)=-\delta
\end{aligned}
$$

which can be reduced to:

$$
\begin{gathered}
\sum_{n=1,3,5, . .}^{\infty} \frac{1}{n} \sin \left(\frac{n \pi}{4}\right) \cos \left(\frac{n \pi}{4}\right) R_{o l}\left(n w_{o}\right)=-\frac{\pi}{8} \\
\sum_{n=1,3,5, . .}^{\infty} \frac{1}{n} \sin ^{2}\left(\frac{n \pi}{4}\right) I_{o l}\left(n w_{o}\right)=-\frac{\pi}{8}
\end{gathered}
$$

The previous equations can be written as:

$$
R_{o l}\left(w_{o}\right)=-\frac{\pi}{4}-\sum_{n=3,5, . .}^{\infty} \frac{2}{n} \sin \left(\frac{n \pi}{4}\right) \cos \left(\frac{n \pi}{4}\right) R_{o l}\left(n w_{o}\right)
$$

and 


$$
I_{o l}\left(w_{o}\right)=-\frac{\pi}{4}-\sum_{n=3,5, . .}^{\infty} \frac{2}{n} \sin ^{2}\left(\frac{n \pi}{4}\right) I_{o l}\left(n w_{o}\right)
$$

As described in section 4, the critical point $\mathrm{C}$ to avoid oscillations is located in $-\frac{\pi}{4}-\frac{\pi}{4} j$. If the frequency response of the open loop were zero for higher order harmonics, then the previous condition would recall the one derived from describing function analysis. Therefore, the summation terms in equations (27) and (26) represent the effect of the higher order harmonics on the oscillation condition defined by the point $\mathrm{C}$. The exact frequency of oscillation, $w_{o}$, for which equations (27) and (26) are true, is difficult to obtain. However, if the summation terms are small, it will be closed to the frequency $w_{c}$ where $G_{o l}(j w)$ crosses the point $C=-\frac{\pi}{4}-\frac{\pi}{4} j$. Therefore, in order to define a simple criterion to evaluate the effect of higher order harmonics, a frequency $w_{o}=w_{c}$ will be considered to calculate a new point in the complex plane, $C^{\prime}$, by applying equations (27) and (26). As can be noted, the effect of the $n^{\text {th }}$ harmonic is attenuated by the coefficient $\frac{1}{n}$, so the contribution of the harmonics decrease significantly with $n$. Taking this into account, in the following we only consider the effect of the $3^{\text {th }}, 5^{\text {th }}$ and $7^{\text {th }}$ harmonics: harmonics for $n>7$ are neglected. Under this assumption, the new point that approximately defines the oscillation condition is reduced to:

$$
\begin{gathered}
R e\left(C^{\prime}\right)=-\frac{\pi}{4}+\frac{1}{3} R_{o l}\left(3 w_{c}\right)-\frac{1}{5} R_{o l}\left(5 w_{c}\right)+\frac{1}{7} R_{o l}\left(7 w_{c}\right) \\
\operatorname{Im}\left(C^{\prime}\right)=-\frac{\pi}{4}-\frac{1}{3} I_{o l}\left(3 w_{c}\right)-\frac{1}{5} I_{o l}\left(5 w_{c}\right)-\frac{1}{7} I_{o l}\left(7 w_{c}\right)
\end{gathered}
$$

The limit cycle will appear if the closed loop transfer function $G_{o l}(j w)$ encircles the new point $C^{\prime}$. Therefore, the distance from that point to the original one, $C$, is a measure of the validity of the describing function approach. A convenient way to measure that distance, from the robustness point of view is by defining the difference in phase in the following way. Let us define $\Delta \Phi_{\text {ssod }}$, as the difference in phase of the open loop transfer function between the point where $\left|G_{o l}(j w)\right|=\left|C^{\prime}\right|$, and the point $C^{\prime}$, as shown in Figures 8 and 9. If this difference is negative, it means that the system will oscillate even if it does not cross the $C$ point, i.e. the higher order harmonics decrease the robustness to limit cycles. If this difference is positive, it means that the system will not oscillate even if it crosses the $C$ point, i.e., the higher order harmonics increase the robustness to limit cycles. This fact is illustrated in the next section, where an extensive simulation study is presented. 


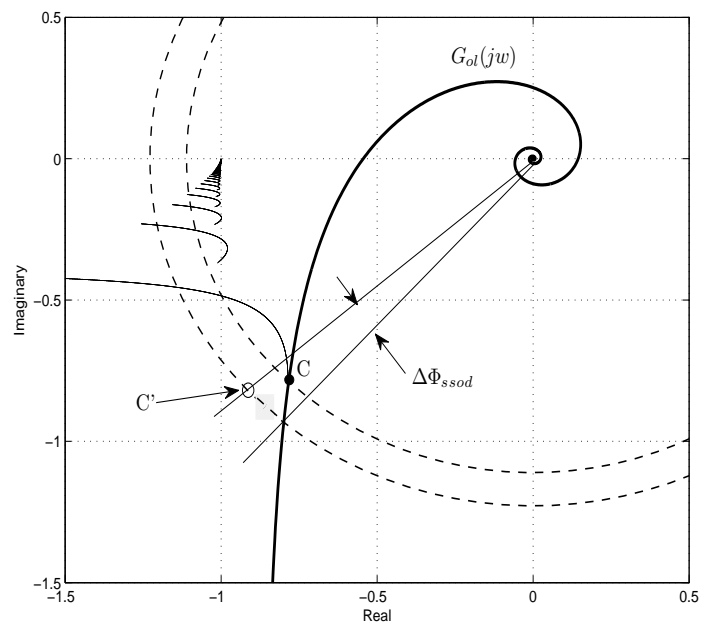

Figure 8: Higher order harmonics contribution to robustness, $\Delta \Phi_{\text {ssod }}$. Positive case.

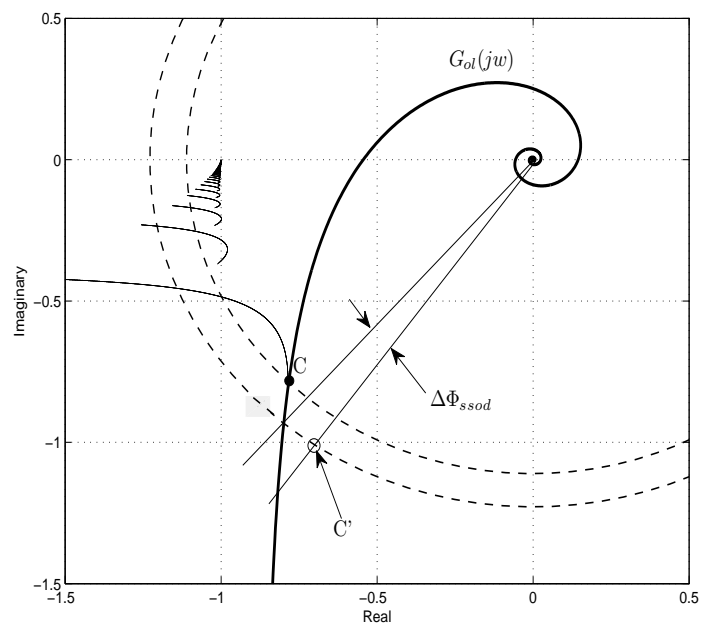

Figure 9: Higher order harmonics contribution to robustness, $\Delta \Phi_{\text {ssod }}$. Negative case. 


\section{Simulation study}

In order to verify the applicability of the proposed method, a well known batch of models (30a)-(30e) that summarizes the most common dynamics encountered in industrial processes is used, [19].

$$
\begin{gathered}
G_{1}(s)=\frac{e^{-5 s}}{(s+1)^{3}} \\
G_{2}(s)=\frac{9}{(s+1)\left(s^{2}+2 s+9\right)} \\
G_{3}(s)=\frac{1}{(s+1)^{4}} \\
G_{4}(s)=\frac{1}{(s+1)^{7}} \\
G_{5}(s)=\frac{1-2 s}{(s+1)^{3}}
\end{gathered}
$$

Table 1 shows the values of $\Delta \Phi_{\text {ssod }}$ for all the studied models. In all cases $\Delta \Phi_{\text {ssod }}>0$, therefore the effect of the higher order harmonics is always to increase the robustness to limit cycle respect to the results obtained with the DF technique. The values in columns two and three are the effect of the higher harmonics in the point $C$, that is:

$$
\begin{aligned}
\Delta C_{R} & =\frac{1}{3} R_{o l}\left(3 w_{c}\right)-\frac{1}{5} R_{o l}\left(5 w_{c}\right)+\frac{1}{7} R_{o l}\left(7 w_{c}\right) \\
\Delta C_{I} & =-\frac{1}{3} I_{o l}\left(3 w_{c}\right)-\frac{1}{5} I_{o l}\left(5 w_{c}\right)-\frac{1}{7} I_{o l}\left(7 w_{c}\right)
\end{aligned}
$$

\begin{tabular}{|c|c|c|c|}
\hline Model & $\Delta C_{R}$ & $\Delta C_{I}$ & $\Delta \Phi_{\text {ssod }}($ degree $)$ \\
\hline$G_{1}$ & -0.089 & -0.095 & 4.4 \\
\hline$G_{2}$ & -0.049 & -0.067 & 1.2 \\
\hline$G_{3}$ & -0.039 & -0.050 & 2.4 \\
\hline$G_{4}$ & -0.068 & -0.075 & 3.5 \\
\hline$G_{5}$ & -0.097 & -0.112 & 4.7 \\
\hline
\end{tabular}

Table 1: Values of $\Delta \Phi_{\text {ssod }}$ for systems $G_{1}$ to $G_{5}$ 
For all these systems SSOD based PI controllers were designed applying the tuning method proposed in the previous section, with $\phi_{m, s s o d}=10 \mathrm{deg}$. Figure 10 shows the polar plots obtained with the PI controllers. It can be noted that all cases fulfill the robustness conditions $\phi_{m, s s o d}=10^{\circ}$ and $\gamma_{m} \geq 2$, therefore limit cycle oscillations should not appear in the closed loop control systems. This fact is confirmed in Figures 11 and 12 where the responses for time-driven and SSOD-based controllers are depicted. For the simulation of the time-driven controller, fixed sampling periods $T=0.3,0.05,0.1,0.2,0.2 \mathrm{sec}$ have been used for systems $G_{1}(s)-G_{5}(s)$ respectively. For the simulations of the SSOD-based controllers the value of $\delta=0.1$ has been considered. The instants when events take place with this value of $\delta$ are also presented in the figures.

As can be noted the responses of conventional and SSOD-based controllers are similar despite the significant reduction of required measurement transmissions in the event based approach. In order to show that the oscillations really appear if the new robustness measure is not ensured, PI controllers have been designed for $\phi_{m, s s o d}=-10^{\circ}$ and $\gamma_{m} \geq 2$. In Figure 13 the polar plots obtained for those PI controllers are shown. All the Nyquist plots intersect the DF plot, predicting the oscillations. Figure 14 shows the simulations results that confirm the persistent oscillations that appear in the time response.

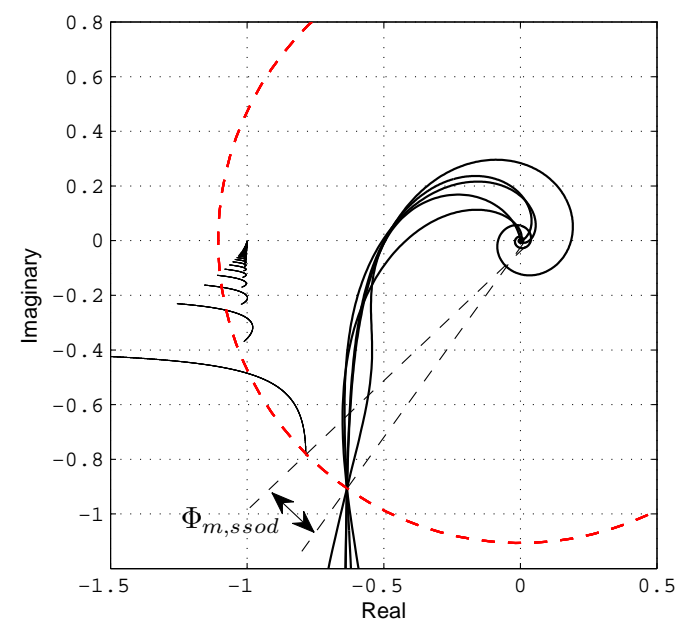

Figure 10: Polar plot of $-1 / N$ and the open loop transfer functions for PI controller designed for systems $G_{1}(s)-G_{5}(s)$ with $\phi_{m, \text { ssod }}=10^{\circ}$. 

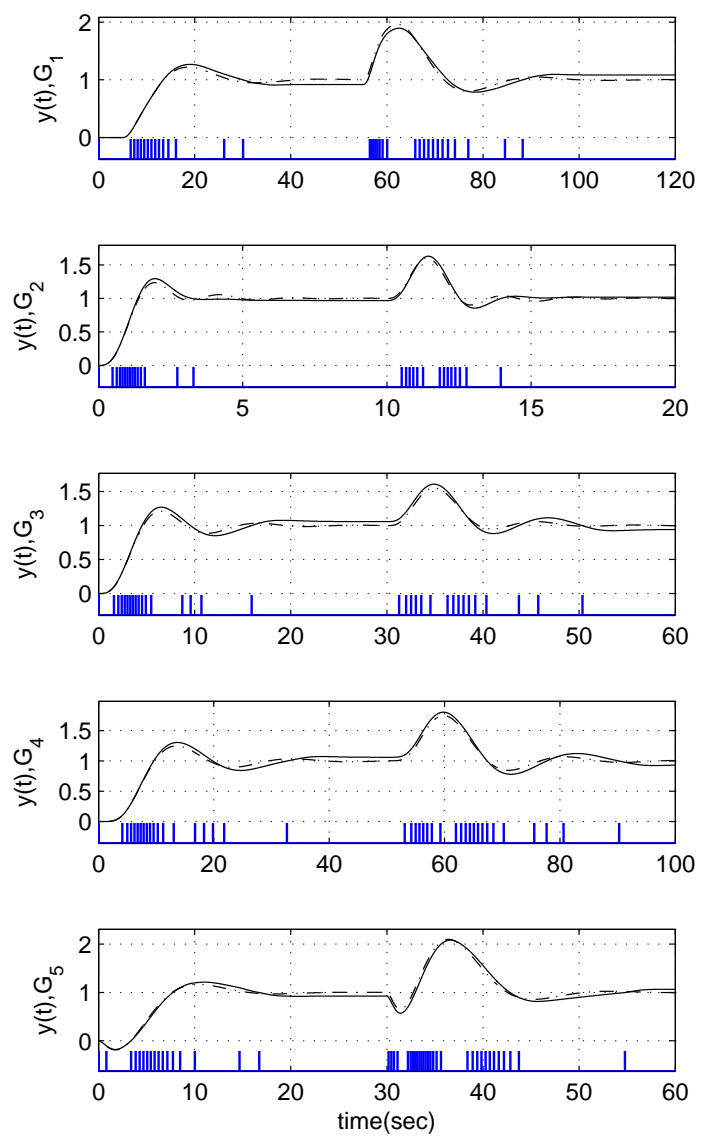

Figure 11: Time response of the controlled outputs to step-like changes in reference and disturbance of magnitude 1 for the systems $G_{1}(s)-G_{5}(s)$ with PI controllers with $\phi_{m, s s o d}=10^{\circ}$. Dashed line: time-driven controllers. Solid line: SSOD-based controllers with $\delta=0.1$. Vertical lines: events. 

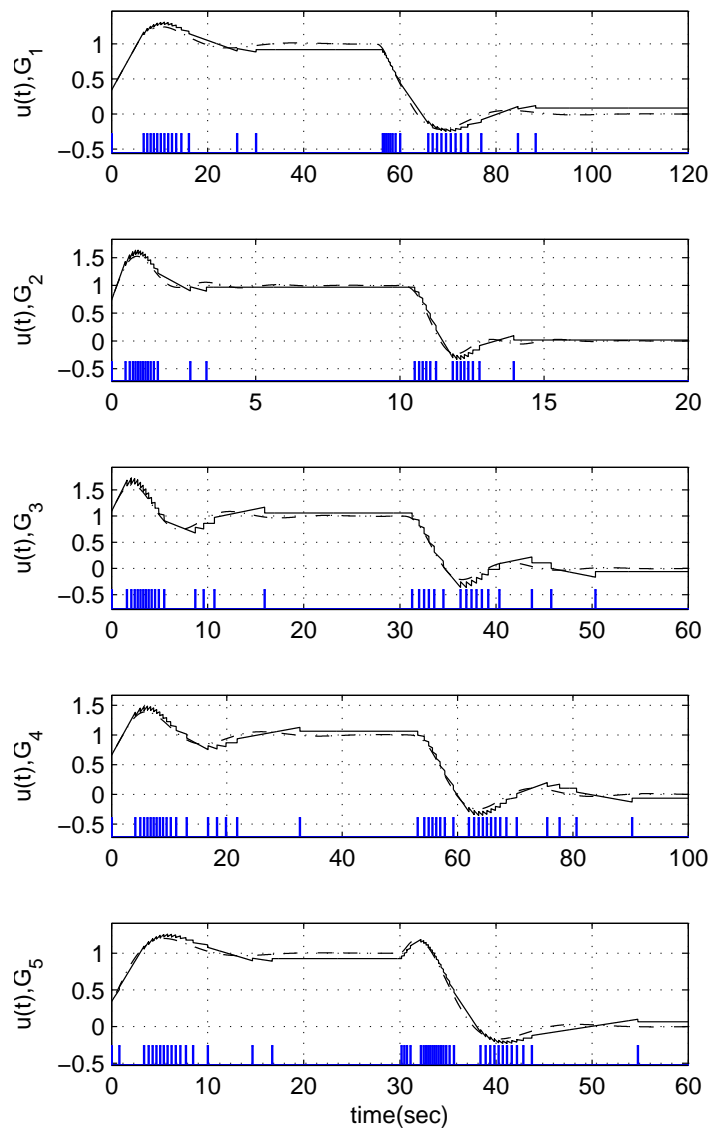

Figure 12: Time response of the control actions to step-like changes in reference and disturbance of magnitude 1 for the systems $G_{1}(s)-G_{5}(s)$ with SSOD based PI controllers with $\phi_{m, s s o d}=10^{\circ}$ and $\delta=0.1$. Vertical lines: events. 


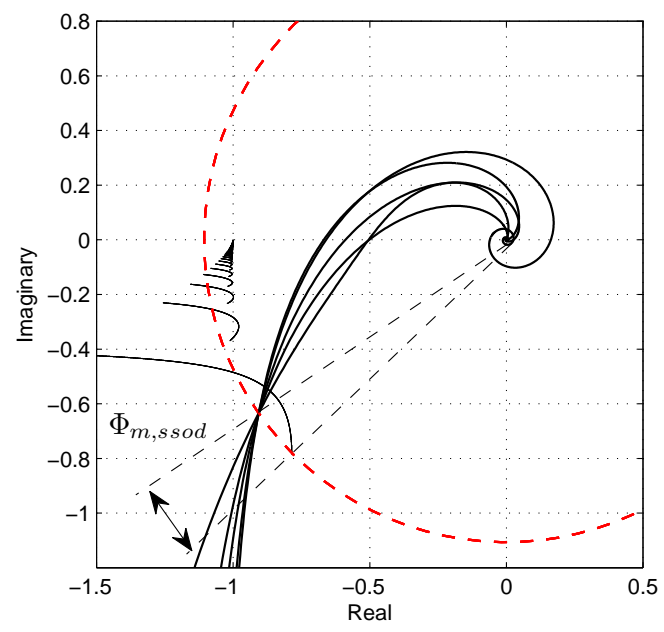

Figure 13: Polar plot of $-1 / N$ and the open loop transfer functions for PI controller designed for systems $G_{1}(s)-G_{5}(s)$ with $\phi_{m, s s o d}=-10^{\circ}$

\section{Java based design tool}

An application has been developed in Java to implement the PI design strategy and to simulate the response of the controlled system to setpoint and disturbance changes. The tool can be downloaded from https://sites.google.com/a/uji.es/freepidtools/sendon-delta-pi-design-tool. The tool allows to define the desired SOD phase margin and the minimum required gain margin, and to select the value of parameter $a$ with a simple slider, and calculates the controller for that value of $a$ (applying equations (10) and (12)). In addition, the optimum PI controller (solution of optimization problem (14)) can be calculated by simply pressing the button "Maximize Ki". Furthermore, if "Auto Maximize Ki" is checked, every time the phase margin is changed through the slider, the optimum controller is automatically calculated. The included output response simulator allows changing the value of SOD parameter $\delta$ through a slider, making very easy to analyze the effect of $\delta$ on the behavior and the number of events. The SSOD-PI scheme is used by default, but the PI-SSOD scheme can also be simulated (with or without dead band). It also allows to simulate the effect of a digital controller implementation with the desired sampling period. Figure 16 shows the main window of the application and the time response simulation window. 

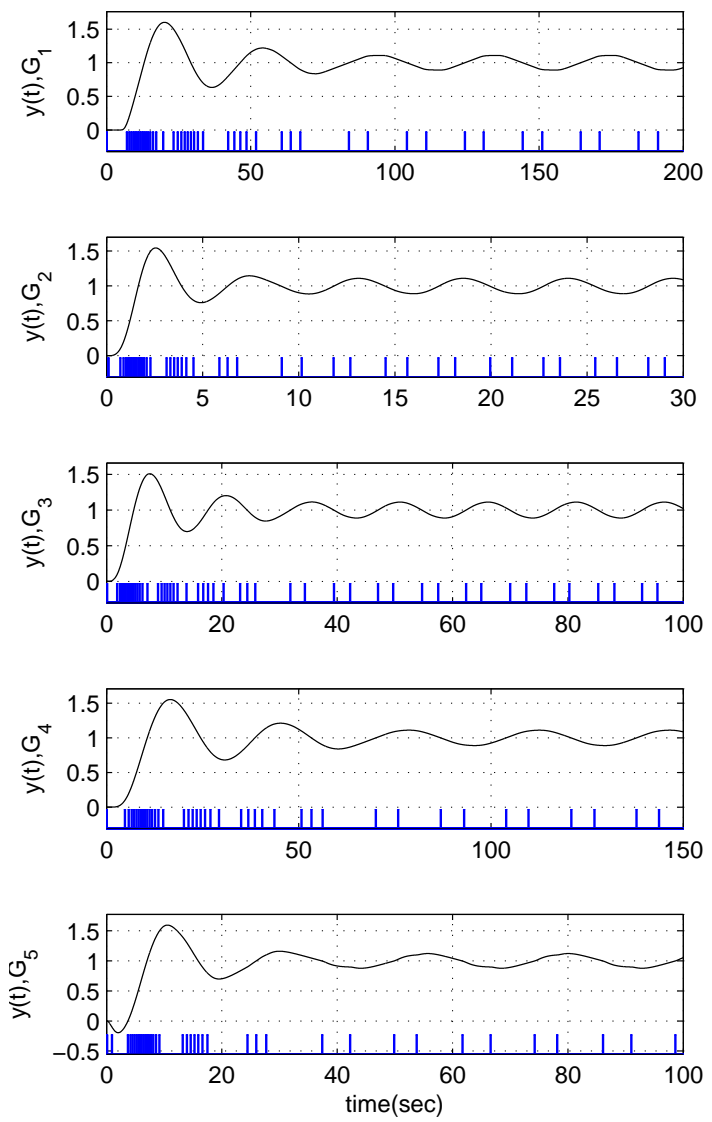

Figure 14: Time response of the controlled outputs to step-like changes in reference of magnitude 1 for the systems $G_{1}(s)-G_{5}(s)$ with SSOD-based PI controllers with $\phi_{m, s s o d}=-10^{\circ}$ and $\delta=0.1$. Vertical lines: events. 

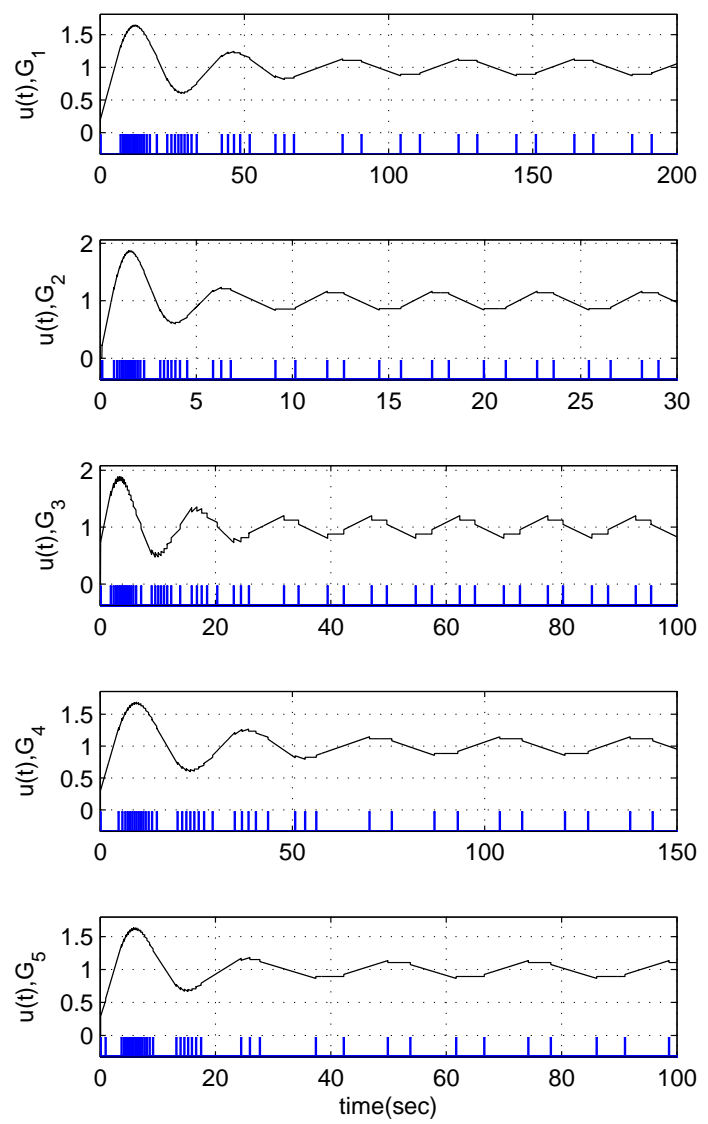

Figure 15: Time response of the control actions to step-like changes in reference of magnitude 1 for the systems $G_{1}(s)-G_{5}(s)$ with SSOD-based PI controllers with $\phi_{m, s s o d}=-10^{\circ}$ and $\delta=0.1$. 

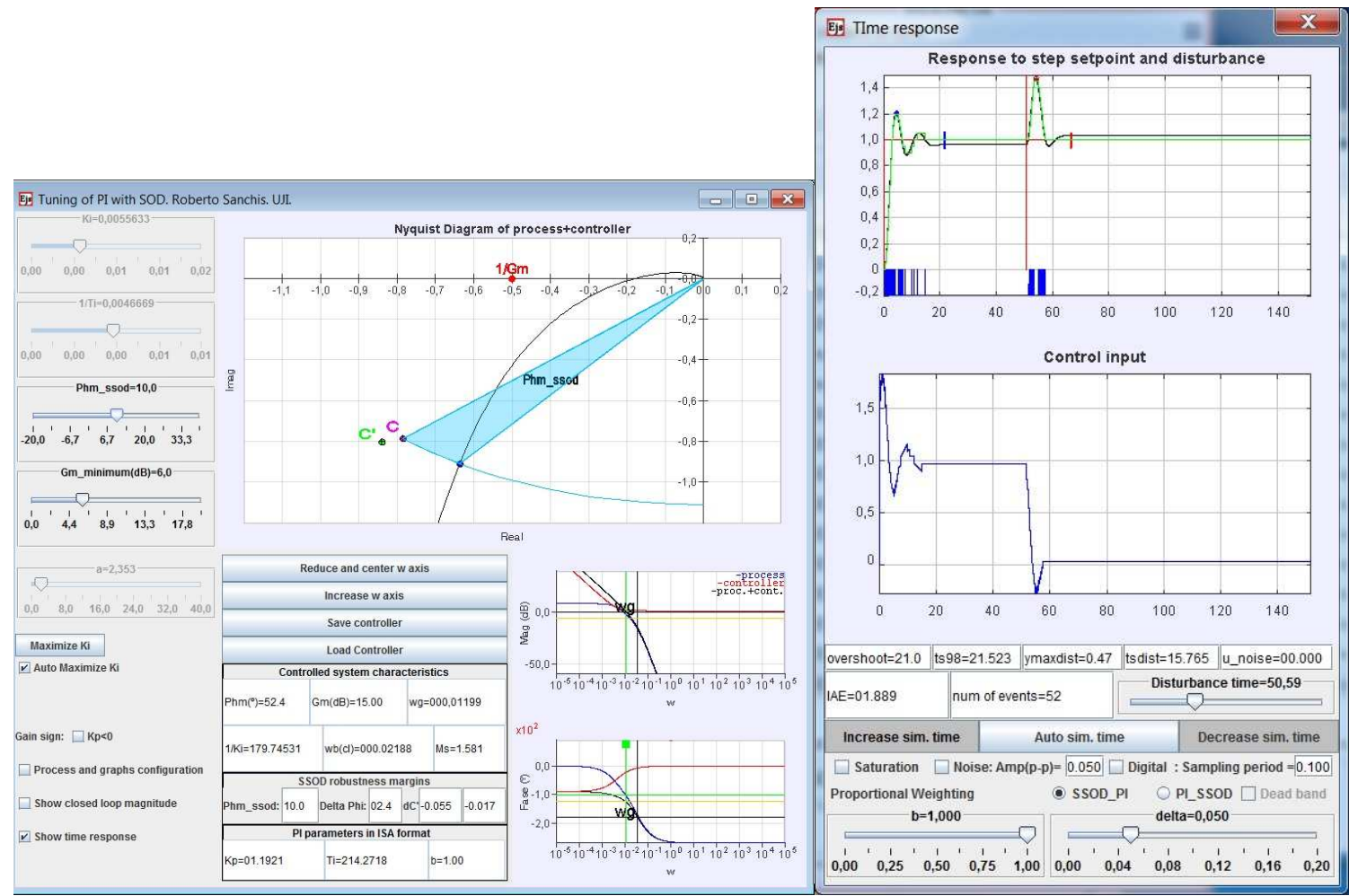

Figure 16: Java application interface: main window (left) and time response simulation window (right). 


\section{Experimental Validation}

In order to verify the reliability of the proposal, the tuning method was used to design the SSOD based PI for an electro-thermal system. The process to be controlled is composed by a heater resistor attached to an iron plate inside a plastic cover. A switched amplifier drives the resistor, while a thermocouple with an amplifier is used to measure the temperature. The system is completed by a small fan that can be used to apply a disturbance to the plant. A picture of the complete electro-thermal system (without the plastic cover) is presented in Figure 17.

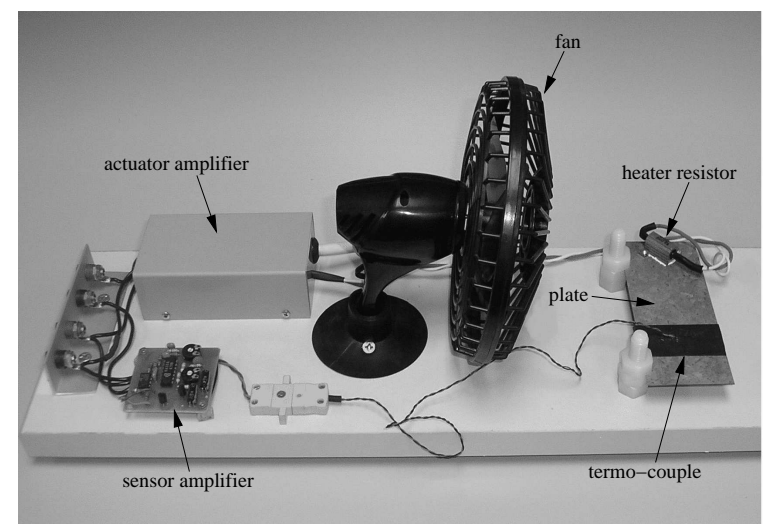

Figure 17: Picture of the electro-thermal process used for the experiment

The event based control system in Figure 1 was implemented using two Modicom340 PLC with CPU BMX P34 2010 linked through a MODBUS RTU RS485 network with transmission speed of $38400 \mathrm{bits} / \mathrm{sec}$. The implementation scheme is depicted in Figure 18. The PLC actuating as sensor node has a 4 channel analog input module BMX AMI 0410 where the output of the sensor amplifier is connected. On the other hand, the controller/actuator PLC has an 8 digital input/output module BMX DDM 16022, through which the control action is applied to the actuator amplifier as a PWM signal. Two periodic tasks were defined in both the sensor node and the controller/actuator node respectively. The SENSOR task, which runs with period of 100 msec., sends the values of the error according to the SSOD strategy to the PLC where the CONTROLLER task is executed. In the CONTROLLER task a simple Euler discretization of a PI controller with anti windup was implemented running with period of 1 sec..

The transfer function of the electro-thermal system, equation (33), was obtained from the time response of the iron plate temperature to a step like voltage change 


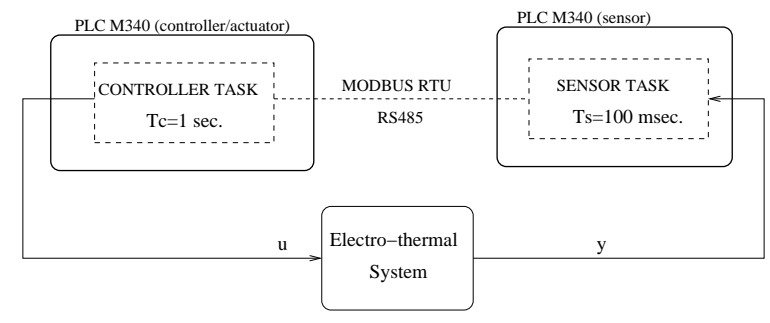

Figure 18: Control scheme used for the practical validation of the proposal.

\begin{tabular}{|c|c|c|c|c|}
\hline$\Phi_{m, \text { ssod }}$ & $\Delta \Phi_{\text {ssod }}$ & $\gamma_{m}(d B)$ & $K_{p}$ & $T_{i}$ \\
$-10^{\circ}$ & $2.4^{o}$ & 10 & 1.84 & $146.06 \mathrm{sec}$. \\
$10^{\circ}$ & $2.4^{o}$ & 15 & 1.19 & $214.21 \mathrm{sec}$. \\
\hline
\end{tabular}

Table 2: PI design results for the electro-thermal system.

applied to process input. Two PI controllers were designed using this transfer function according to the method addressed in section 4.1 for $\Phi_{m, s s o d}=-10^{\circ}$ and $\Phi_{m, s s o d}=10^{\circ}$, both with $\gamma_{m} \geq 8 d B$. The controllers were designed with the Java application described in section 7 . Figures 19 and 20 show the resulting design for $\Phi_{m, s s o d}=-10^{\circ}$ and $\Phi_{m, s s o d}=+10^{\circ}$ respectively. The most important characteristics of the designs are summarized in Table 8.

$$
G(s)=\frac{2.66}{(1+26.5 s)^{2}(1+244 s)}
$$

According to the values of $\Phi_{m}$ and $\gamma_{m}$, stable closed loop responses should be obtained for both controllers when implemented in a time-driven approach with a properly selected sample time. However, if SSOD sampling strategy is used, the controller designed with $\Phi_{m, s s o d}=-10^{\circ}$ will induce limit cycle oscillations, as predicted in this article. This fact is confirmed by the actual system response which is shown in Figure 21, where the response to a step change in the setpoint (at $t=460$ seconds) and to a step change in the disturbance (at $t=1700$ seconds) are shown. It can be noted that for time greater than 3000 sec., after the disturbance correction, the system response presents limit cycle oscillations, which can be seen in more detail in Figure 22.

On the other hand, the controller designed with $\Phi_{m, s s o d}=10^{\circ}$ produces a stable response without limit cycles, as is shown in Figure 23. It is worth highlighting that in the actual application, as well as in simulations presented in section 6 , the close loop response of the SSOD based system is similar to that of the time-driven case, 

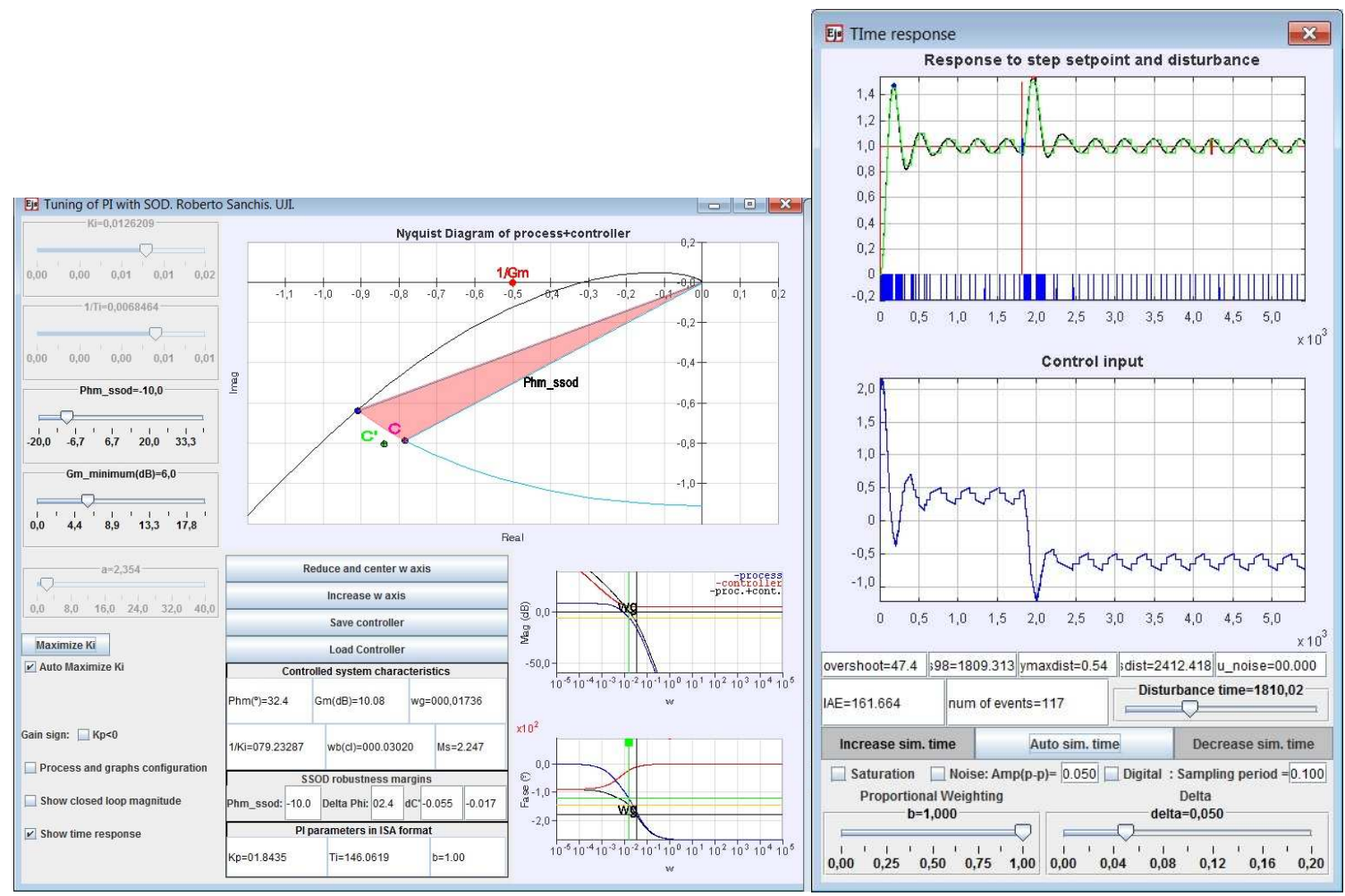

Figure 19: Java application interface: results for the design with $\Phi_{\text {ssos }}=-10^{\circ}$. 

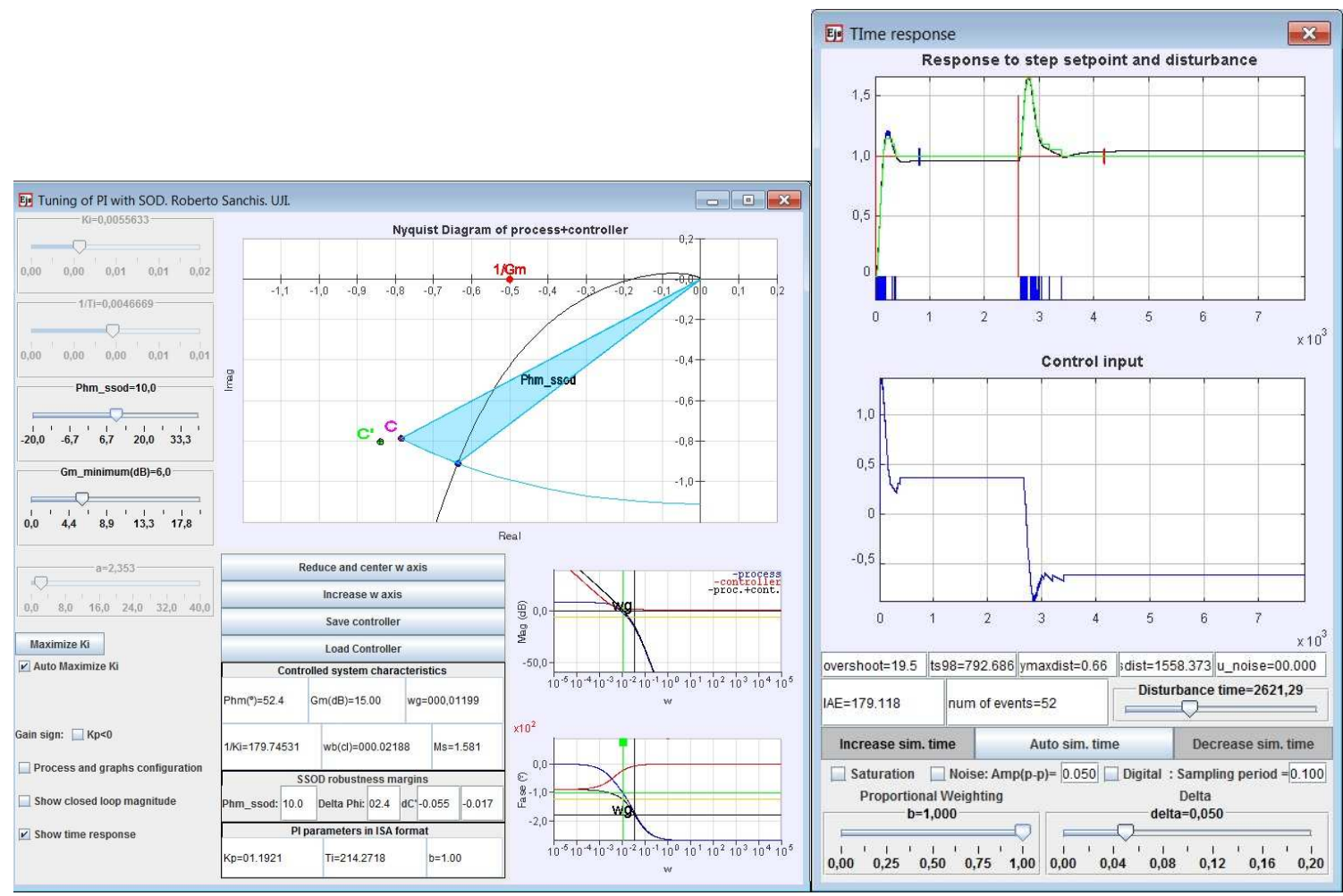

Figure 20: Java application interface: results for the design with $\Phi_{\text {ssos }}=+10^{\circ}$. 
which has been obtained here by continuous simulation using the model (33) and is presented in Figure 24.
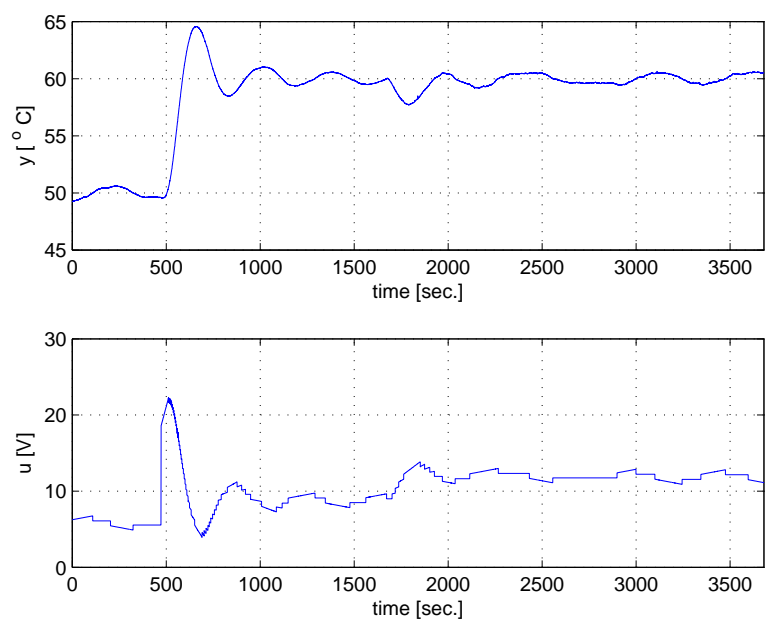

Figure 21: Time response of the controlled outputs to step-like changes in reference and disturbance for the electro-thermal system with PI controllers with $\phi_{m, s s o d}=-10^{\circ}$ and $\delta=0.5^{\circ} \mathrm{C}$.

\section{Conclusions}

In this paper we have analyzed the conditions that produce oscillations in event based PI controllers, assuming a symmetric send on delta scheme. The problem is addressed by means of the describing function (DF). A critical point has been found in the DF plot that determines the existence of oscillations. Conditions for oscillation are reduced to the encirclement of that point by the Nyquist plot. Taking this into account, a new method for tuning SSOD based PI controller is presented to avoid the limit cycles, including the introduction of a new robustness to oscillations performance measure.

The effect of higher order harmonics in the tuning method has also been studied. Surprisingly, it has been found that, for most systems, the contribution of higher order harmonics does not impose further restrictions to the tuning method. Instead, it provides an additional margin to avoid oscillations, that increases the one imposed by the DF method.

Another interesting conclusion is that the appearance of limit cycles does not depend on the value of $\delta$, even if the higher order harmonics are considered. The 

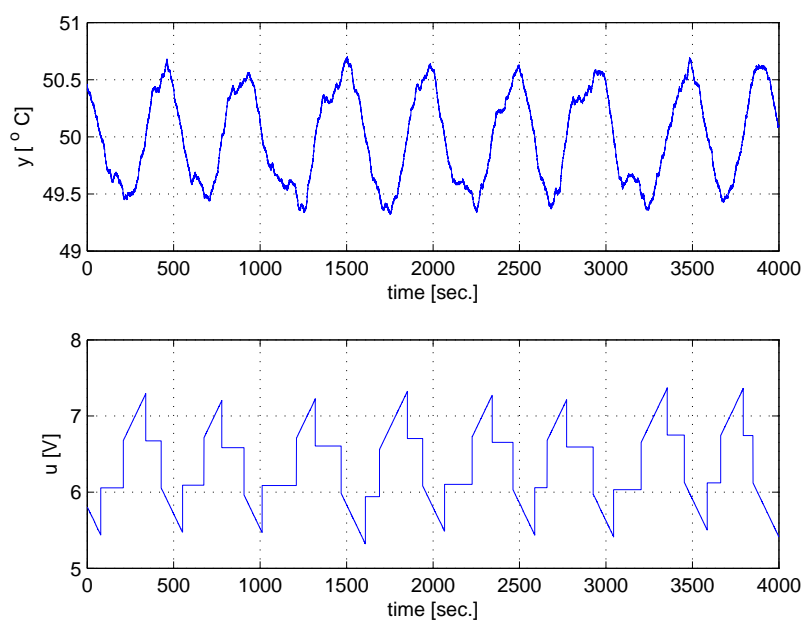

Figure 22: Temperature oscillations in the electro-thermal system with PI controllers with $\phi_{m, s s o d}=$ $-10^{\circ}$ and $\delta=0.5^{\circ} \mathrm{C}$
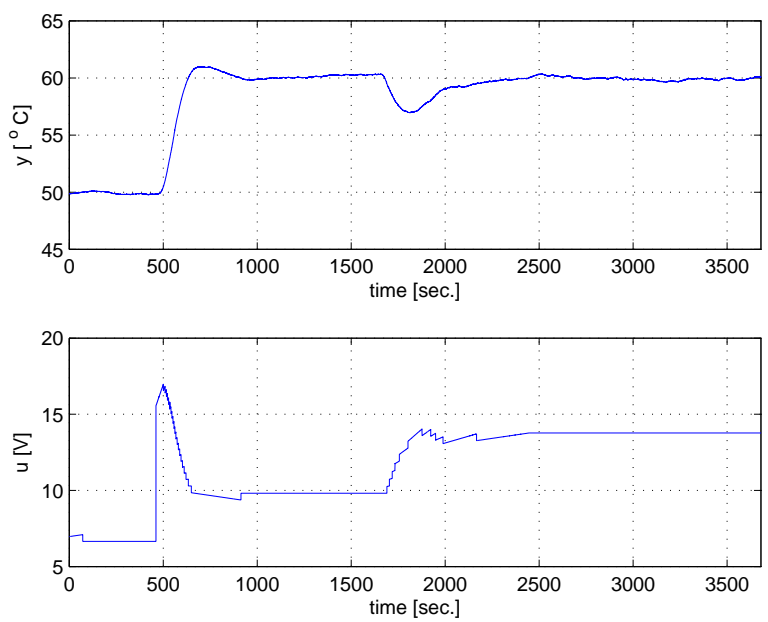

Figure 23: Time response of the controlled outputs to step-like changes in reference and disturbance for the electro-thermal system with PI controllers with $\phi_{m, s s o d}=10^{\circ}$ and $\delta=0.5^{\circ} \mathrm{C}$. 

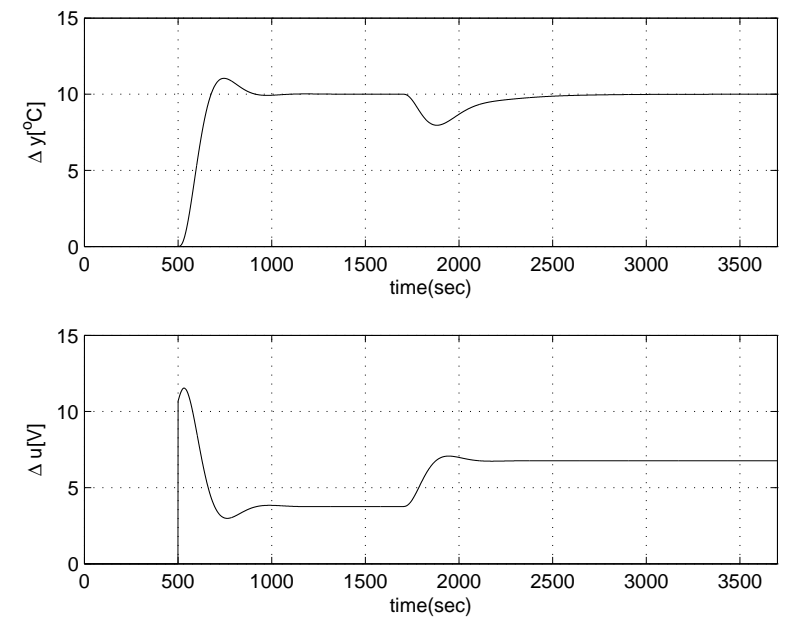

Figure 24: Time response of the controlled output and the control action to step-like changes in reference and disturbance obtained by continuous simulation considering the model (33) and the PI controllers designed with $\phi_{m, s s o d}=10^{\circ}$.

value of $\delta$ affects the amplitude of oscillations (if they appear), the steady state error and the number of events (measurements transmission).

The proposed ideas are illustrated in simulations with a batch of models widely used in the literature to test PI design methods. The feasibility of the proposal has been also corroborated by the application to the control of an actual laboratory scale electro-thermal system. Additionally, the tuning method has been implemented in an easy to use Java based application (https://sites.google.com/a/uji.es/freepidtools/sendon-delta-pi-design-tool), which was developed to aid the design and simulation of SSOD based PI controllers.

\section{Acknowledgements}

This work has been supported by the MICINN under grant DPI2011-27845-C0202.

\section{Appendix A. Describing Function of the SSOD_ZOH sampler}

The output equation of SSOD_ZOH sampler, whose input/output relation is presented in Figure 2, is: 


$$
\bar{e}(t)= \begin{cases}(i+1) \delta & \text { if } e(t) \geq(i+1) \delta \text { and } \bar{e}\left(t^{-}\right)=i \delta, i \in \mathbb{Z} \\ (i-1) \delta & \text { if } e(t) \leq(i-1) \delta \text { and } \bar{e}\left(t^{-}\right)=i \delta \\ i \delta & \text { if } e(t) \in[(i-1) \delta,(i+1) \delta] \text { and } \bar{e}\left(t^{-}\right)=i \delta\end{cases}
$$

For a sinusoidal input $e(\phi)=A \sin (\phi)$, the output of the SSOD_ZOH block can be expressed as

$$
\begin{aligned}
\bar{e}(\phi) & =\delta \sum_{k=1}^{i} \operatorname{sgn}\left(\left.\frac{d e(\phi)}{d \phi}\right|_{\phi_{k}}\right) \quad \forall \phi ; \phi_{i}<\phi<\phi_{i+1} \\
& =\delta \sum_{k=1}^{i} \operatorname{sgn}\left(\cos \phi_{k}\right) \quad \forall \phi ; \phi_{i}<\phi<\phi_{i+1}
\end{aligned}
$$

where

$$
\operatorname{sgn}(e)= \begin{cases}1 & \text { if } e>0 \\ -1 & \text { if } e<0 \\ 0 & \text { if } e=0\end{cases}
$$

The SSOD_ZOH sampler is an odd no-linearity where the history of the input determines the value of the output in the multiple-valued regions. The describing function for this kind of nonlinearity can be calculated as:

$$
\begin{aligned}
N(A) & =\frac{2 j}{\pi A} \int_{0}^{\pi} \bar{e}(\phi) \exp (-j \phi) d \phi \\
& =\frac{2 j}{\pi A}\left(\int_{\phi_{1}}^{\phi_{2}} \operatorname{sgn}\left(\cos \phi_{1}\right) \delta \exp (-j \phi) d \phi+\right. \\
& \int_{\phi_{2}}^{\phi_{3}}\left(\operatorname{sgn}\left(\cos \phi_{1}\right)+\operatorname{sgn}\left(\cos \phi_{2}\right)\right) \delta \exp (-j \phi) d \phi+\ldots \\
& \ldots+\int_{\phi_{i}}^{\phi_{i+1}} \sum_{k=1}^{i}\left(\operatorname{sgn}\left(\cos \phi_{k}\right)\right) \delta \exp (-j \phi) d \phi+\ldots \\
& \left.\ldots+\int_{\phi_{n}}^{\pi} \sum_{k=1}^{n}\left(\operatorname{sgn}\left(\cos \phi_{k}\right)\right) \delta \exp (-j \phi) d \phi\right)
\end{aligned}
$$


Applying the addition of integration on intervals, the previous equation can be rewritten as

$$
\begin{aligned}
N(A) & =\frac{2 D j}{\pi A}\left(\int_{\phi_{1}}^{\pi} \operatorname{sgn}\left(\cos \phi_{1}\right) \exp (-j \phi) d \phi+\right. \\
& \int_{\phi_{2}}^{\pi} \operatorname{sgn}\left(\cos \phi_{2}\right) \exp (-j \phi) d \phi+\ldots \\
& \ldots+\int_{\phi_{i}}^{\pi} \operatorname{sgn}\left(\cos \phi_{i}\right) \exp (-j \phi) d \phi+\ldots \\
& \left.\ldots+\int_{\phi_{n}}^{\pi} \operatorname{sgn}\left(\cos \phi_{n}\right) \exp (-j \phi) d \phi\right) \\
& =\frac{2 D j}{\pi A} \sum_{k=1}^{n}\left(\operatorname{sgn}\left(\cos \phi_{k}\right) \int_{\phi_{k}}^{\pi} \exp (-j \phi) d \phi\right)
\end{aligned}
$$

Taking into account that

$$
\int_{\phi_{k}}^{\pi} \exp (-j \phi) d \phi=-j\left(1+\exp \left(-j \phi_{k}\right)\right)
$$

and

$$
\exp \left(-j \phi_{k}\right)=\cos \phi_{k}-j \sin \phi_{k}
$$

the Equation (A.4) results in

$$
N(A)=\frac{2 \delta}{\pi A}\left(\sum_{k=1}^{n} \operatorname{sgn}\left(\cos \phi_{k}\right)\left(1+\cos \phi_{k}\right)+j \sum_{k=1}^{n} \operatorname{sgn}\left(\cos \phi_{k}\right) \sin \phi_{k}\right)
$$

Since for $m=\left\lfloor\frac{A}{\delta}\right\rfloor \Rightarrow \phi_{m} \leq \pi / 2<\phi_{m+1}$ the following identity is fulfilled

$$
\cos \phi_{m-k}=-\cos \phi_{m+k}, \quad k=1 \cdots m-1,
$$

the terms in the right hand side of Equation (A.5) are transformed to

$$
\sum_{k=1}^{n} \operatorname{sgn}\left(\cos \phi_{k}\right)\left(1+\cos \phi_{k}\right)=1+\cos \phi_{m}+2 \sum_{k=1}^{m-1} \cos \phi_{k}
$$

and 


$$
\sum_{k=1}^{n} \operatorname{sgn}\left(\cos \phi_{k}\right) \sin \phi_{k}=\sin \phi_{m}
$$

Therefore, Equation (A.5)can be rewritten as follows

$$
N(A)=\frac{2 \delta}{\pi A}\left(1+\cos \phi_{m}+2 \sum_{k=1}^{m-1} \cos \phi_{k}-j \sin \phi_{m}\right)
$$

The previous expression for $N(A)$ only depends on $\phi_{k} \leq \pi / 2$, so the following identities can be used to write de describing function in terms of the SSOD_ZOH nonlinearity parameter $\delta$.

$$
\begin{gathered}
\sin \phi_{k}=k \frac{\delta}{A} \Rightarrow \phi_{k}=\arcsin \left(k \frac{\delta}{A}\right) \\
\cos \phi_{k}=\sqrt{1-\left(k \frac{\delta}{A}\right)^{2}}
\end{gathered}
$$

Using equations (A.10) and (A.11) the describing function $N(A)$ can be rewritten as follows:

$$
N(A, \delta)=\frac{2 \delta}{\pi A}\left[1+\sqrt{1-\left(m \frac{\delta}{A}\right)^{2}}+2 \sum_{k=1}^{m-1} \sqrt{1-\left(k \frac{\delta}{A}\right)^{2}}\right]-j m \frac{2 \delta^{2}}{\pi A^{2}}
$$

\section{References}

[1] L. Feeney, M. Nilsson, Investigating the energy consumption of a wireless network interface in an ad hoc networking environment, in: INFOCOM 2001. Twentieth Annual Joint Conference of the IEEE Computer and Communications Societies. Proceedings. IEEE, Vol. 3, 2001, pp. 1548-1557 vol.3. doi:10.1109/INFCOM.2001.916651.

[2] M. Miskowicz, Send-on-delta concept: An event-based data reporting strategy, Sensors 6 (1) (2006) 49-63.

[3] S. Dormido, J. Sanchez, E. Kofman, Muestreo, control y comunicacion basados en eventos, Revista Iberoamericana de Automatica e Informatica Industrial RIAI 5 (1) (2008) $5-26$. 
[4] J. Ploennigs, V. Vasyutynskyy, K. Kabitzsch, Comparative study of energyefficient sampling approaches for wireless control networks, Industrial Informatics, IEEE Transactions on 6 (3) (2010) 416 -424. doi:10.1109/TII.2010.2051812.

[5] K.-E. Årzén, A Simple Event-based PID Controller, in: Proc. 14th World Congress of IFAC, Beijing, Vol. Q, 1999, pp. 423-428.

[6] S. Durand, N. Marchand, An Event-Based PID Controller With Low Computational Cost, in: L. Fesquet, B. Torrésani (Eds.), 8th International Conference on Sampling Theory and Applications (SampTA'09), 2009.

URL http://hal .archives-ouvertes.fr/hal-00393031

[7] S. Durand, N. Marchand, Further Results on Event-Based PID Controller, in: Proceedings of the European Control Conference 2009, Budapest, Hongrie, 2009, pp. 1979-1984.

URL http://hal.archives-ouvertes.fr/hal-00368535

[8] V. Vasyutynskyy, K. Kabitzsch, A comparative study of PID control algorithms adapted to send-on-delta sampling, in: Industrial Electronics (ISIE), 2010 IEEE International Symposium on, 2010, pp. $3373-3379$. doi:10.1109/ISIE.2010.5637997.

[9] V. Vasyutynskyy, K. Kabitzsch, Time constraints in PID controls with send-ondelta, in: G. Juanole, S. H. Hong (Eds.), Fieldbuses and Networks in Industrial and Embedded Systems, Vol. 8, 2009.

[10] M. Beschi, S. Dormido, J. Sanchez, A. Visioli, Characterization of symmetric send-on-delta PI controllers, Journal of Process Control 22 (10) (2012) 19301945. doi:10.1016/j.jprocont.2012.09.005.

URL http://linkinghub.elsevier.com/retrieve/pii/S0959152412002247

[11] J. Chacón, J. Sánchez, A. Visioli, L. Yebra, S. Dormido, Characterization of limit cycles for self-regulating and integral processes with PI control and send-on-delta sampling, Journal of Process Control 23 (6) (2013) 826-838. doi:10.1016/j.jprocont.2013.04.001.

URL http://dx.doi.org/10.1016/j.jprocont.2013.04.001

[12] M. Beschi, A. Visioli, Tuning of symmetric send-on-delta proportionalintegral controllers, IET Control Theory and Applications 8. doi:10.1049/ietcta.2013.0048. 
[13] M. Beschi, S. Dormido, J. Sánchez, A. Visioli, Closed-Loop Automatic Tuning Technique for an Event-Based PI Controller, Industrial \& Engineering Chemistry Research 54 (24) (2015) 6362-6370. doi:10.1021/acs.iecr.5b01024.

[14] M. Beschi, S. Dormido, J. Sanchez, A. Visioli, Stability analysis of symmetric send-on-delta event-based control systems, in: American Control Conference (ACC), 2013, 2013, pp. 1771-1776. doi:10.1109/ACC.2013.6580092.

[15] A. Leva, F. Terraneo, Low power synchronisation in wireless sensor networks via simple feedback controllers: The flopsync scheme, in: American Control Conference (ACC), 2013, 2013, pp. 5017-5022.

[16] A. Gelb, W. E. VanderVelde, Multiple-Input Describing Functions and Nonlinear System Design, New York, NY, USA: McGraw-Hill, 1968.

[17] J. Romero, R. Sanchis, I. Peñarrocha, A simple rule for tuning event-based pid controllers with symmetric send-on-delta sampling strategy, in: Emerging Technologies Factory Automation (ETFA), 2014 IEEE 19th Conference on, 2014.

[18] R. Sanchis, J. A. Romero, P. Balaguer, Tuning of pid controllers based on simplified single parameter optimisation, International Journal of Control 83 (9) (2010) 1785-1798. arXiv:http://dx.doi.org/10.1080/00207179.2010.495162, doi:10.1080/00207179.2010.495162.

URL http://dx.doi.org/10.1080/00207179.2010.495162

[19] H. Panagopoulos, K. J. Astrom, T. Hagglund, Design of PID controllers based on constrained optimization, IEE Proc.-Control Theory Appl. 149 (1) (2002) $32-40$. 